\title{
PARAMETRIC STUDY OF THE ERRORS OBTAINED FROM THE MEASUREMENT OF THE OSCILLATING MOVEMENT OF A BRIDGE USING IMAGE PROCESSING
}

\author{
Ferrer, B..$^{*} ;$ Mas, D. ${ }^{2}$; García-Santos, J.I. ${ }^{1}$; Luzi, G. ${ }^{3}$ \\ ${ }^{1}$ Dept. Civil Engineering, Universidad de Alicante. PO BOX 99, 03080 Alicante (SPAIN) \\ ${ }^{2}$ Ins. Physics Applied to Sciences and Technologies, Universidad de Alicante. PO BOX 99, \\ 03080 Alicante (SPAIN) \\ ${ }^{3}$ Centre Tecnològic de Telecomunicacions de Catalunya (CTTC), Division of Geomatics, Av. \\ Gauss 11, E-08860 Castelldefels, Barcelona, (SPAIN) \\ *Corresponding author: belen.ferrer@ua.es; telephone number: +345903400; fax number: \\ $+34965903464$
}

\begin{abstract}
The movement of a pedestrian bridge retrieved by means of image processing technique has been analysed in this paper. An optical target has been attached to the deck and its oscillation has been tracked with fast cameras. The movement of the bridge has also been measured with a radar interferometer and this result has been taken as the reference signal. Using these data, a parametric study of the errors introduced by the image-based methods has been performed. The influence of some variables in the measurement error such as the distance to the target, the image size, the type of camera or the movement amplitude has been analysed for four different distances, and two types of excitations. Results show that the relative error decreases with the amplitude and the target diameter and it increases with the target distance. Additionally, the maximum relative error obtained in most of the analysed cases is below $10 \%$.
\end{abstract}

Keywords: Displacement measurement, long distance vibrometry, image processing, bridges, radar interferometry

\section{ACKNOWLEDGMENTS}

The authors acknowledge the support of the Generalitat Valenciana through the project PROMETEO II/2015/015 and GV/2015/116 and the University of Alicante through the project GRE13-10 


\section{1.- INTRODUCTION}

Vibration measurement is a key problem to solve in civil structures, such as bridges, tall buildings or historical constructions. Movements due to vibration may damage the structures if the amplitude of movement is high enough or even if the vibration is maintained for long time. These measurements are traditionally performed with accelerometers but, in some occasions, the complexity or accessibility of the specimen to be measured increases the installation cost and may be even hazardous for the test crew safety. This issue has been decisive to encourage the use of non-contact methods for this kind of measurements. Methods based on the image have been intensively developed in the last years thanks to its easy performance and the increased capabilities of imaging systems.

Bridges, among all civil structures, have some particularities that make them very suitable to conduct experiments using new measurement techniques. In many cases, and due to its stiffness, the vibration amplitudes at certain points of the bridge are larger than in other structures that suffer similar excitation forces, which helps the detection. Also, that movement can be imposed, in the case of a very slender bridge, or predicted in time by the pass of loads above, as it happens in bridges for trains. Additionally, it is easy to find some bridges in which the distance from a steady point to the structure is shorter than that found in other structure types. This allows locating measuring devices close to the bridge to make the measurement both with an experimental device and with a contrast system. Hence one can find several papers where image processing measuring techniques have been tested in bridges but not in other civil structures.

Up to our knowledge, one of the first experiments in bridges for assessing its mechanical properties with image processing methods was done by Wahbeh et al. [1] using high-resolution low-power light emitting diodes (LEDs) as targets. A nonlinear Gaussian regression curve was fitted to every LED image in order to determine the centre of the light spot. The excitation of the bridge was produced by the traffic (four lanes) and the maximum movement measured was higher than $25 \mathrm{~mm}$, thus showing that a camera system could be a good system to measure displacement time histories of real infrastructures. 
The use of LEDs requires of precise installation and orientation of the light source so printed plastic or cardboard targets have replaced the initial luminous targets. A non-luminous target formed by four white spots on a black background was used by Lee and Shinozuka [2]. This target was used to measure the movement of a bridge when some trucks with different loadings and velocities were running on the deck. The movement was obtained by counting the shift in the imaged target spots. The procedure measured movement amplitudes larger than $3 \mathrm{~mm}$ from a distance of $70 \mathrm{~m}$. The resolution obtained was of one pixel, which was good enough for this particular case but, as we will show here, could be further optimized.

Regarding the image analysis procedure, one of the most used techniques is correlation; we can find in the literature numerous examples of the use of digital image correlation (DIC) to measure movements. Several authors [3-5] used DIC to measure strain in concrete surfaces of bodies under different types of loading. Cracks in concrete has been also found using DIC [6]. In bridges, correlation, as well as edge detection and template matching has been successfully used to measure the movement of a bridge under the loading due to train pass-by [7]. All these techniques give single pixel accuracy, although sub-pixel accuracy can be implemented without increasing the complexity of the experimental setup.

By convenient choosing the target shape, one can locate the centroid of the target by simple least squares fitting, obtaining subpixel accuracy even with a pocket low-resolution camera, as it was demonstrated by Mas et al. [8]. Measuring the movement from a distance of $10 \mathrm{~m}$ and in laboratory conditions, the error obtained was below $0.1 \mathrm{~mm}$. Using this procedure, the strain in a concrete surface was measured with good agreement with strain gauges measurement [9]. A similar method has been used by Chen et al. [10] to measure the vibration of some stay cables.

At this point we would like to call the reader's attention on the consequences of using compact commercial cameras in tracking tasks. In [9] the tracking performance of a low-cost consumer camera is compared with that from a high resolution camera. Low cost cameras introduce JPEG compression in the video sequence, thus degrading the final quality of the image. There we show that, even with the compression, the camera is able to track a moving target with high precision. 
The JPEG algorithm introduces a high frequency noise and tiling effect in the image borders. The effect on a large image with high contrast is not very important for our purposes, just introducing some distortion in the detected contour that will increase the error of the ellipse fitting, although centroid location will be accurate although noisy.

A different image-based technique was used by Fukuda et al. [11]. In this work an orientation code matching was used to find both the target and any particular part of the bridge used as a "natural" target. This method assigns an orientation angle to each pixel, which is obtained by finding the steepest ascent orientation evaluated from the pixel neighbourhoods. Subpixel precision was obtained by interpolation of the resultant signal, thus resulting in a suboptimal location method. The distance from camera to the bridge was $300 \mathrm{~m}$. It was demonstrated that the orientation code matching allows the measurement without target with a similar accuracy of that obtained using a designed target. Nevertheless, the measurement error was only checked in laboratory tests and therefore the error due to long distance and open air measurement was not determined.

Despite the good results presented, to the best of our knowledge, the error analysis in the literature is very simple and does not include the influence of the different parameters that could affect the final error, such as the camera type, the distance to target, the target size or the movement amplitude, among others. A study made by Busca [7] analysed the variations in error due to the target scaling factor, that is to say, the target size in the image. In this work the authors measured the movement of a bridge using two different cameras and different field of view thus having different scaling factors $(\mathrm{mm} / \mathrm{px})$ obtained by modifying the zoom of the camera. In all tests the same distance to the target was used, therefore, no conclusions regarding the distance to the object were obtained.

Distance from camera to target influences the measurement not only due to the change of spatial resolution for each distance (at maximum zoom) but also due to the atmospheric distortion and the amplification of small camera movements. Atmospheric distortion, also known as seeing, is a blurring effect of the atmosphere that leads to a degradation of the image quality. This degradation 
results from fluctuations in the refractive index of air. One of the consequences is a smoky effect in a sequence of frames. This phenomenon is of fundamental importance in astronomical research since it limits very much the resolution of terrestrial telescopes. It is well known that, even in the best atmospheric conditions (small islands and high altitude), the atmospheric seeing limits the image resolution to about 0.5 arcseconds [12]. For a medium-size urban area this value can reach 2 arcseconds [13]. Those values correspond to the seeing for astronomic view in which the line of sight is almost perpendicular to the Earth. For our purpose the line of sight is parallel to the Earth surface and, in sunny and hot days (best visual conditions) the heat from ground will affect negatively to the image quality. Therefore, the distance to target should be taken into account.

In this work the distance to target has been selected as variable parameter, as well as the size of the target image, the amplitude of movement, the type of camera and even the type of oscillating movement. The movement measured is an oscillation imposed by a swinging or running person on a pedestrian bridge. The image processing technique selected is template matching with least square fit to have subpixel accuracy. On this procedure the target selected has a contour that can be easily described by a theoretical model. To this end, the most appropriate targets are those which preserve the topology at all possible movements. The simplest one that fulfils this characteristic is the ellipse. Notice also that in this method the subpixel accuracy is obtained in the target location in each particular frame, and not by interpolation between two consecutive locations, so the method provides enhanced location of the target. The improvement on the accuracy of the method using an ellipse and three-dimensional rotations was described by Roig et al. [14]. Therefore, a circular shape has been selected as target, because the projection of a circle seen from different points of view is an ellipse with different orientation and axis. Also note that the apparent size of the axis permits obtaining the perspective correction for in-plane distance measurement.

On the image, the target contour is isolated using an edge detection filter, such as a Sobel operator. The result is a pixelated image in which a change of location smaller than one pixel will only affect to a few pixels. That small change leads to an uncertain target location. To improve the 
accuracy, a fit of the pixelated image of the contour to the theoretical model is done. This implies that the location accuracy for each frame is statistically increased. Once the ellipse is located, the centre of it is selected as reference point. The complete description of the procedure has been already done in [8].

In what follows, we will describe the methods and procedures to analyse the vibration of a pedestrian bridge. Measurements will be taken with video cameras and results from the image processing methods will be compared with those obtained from radar interferometry. The materials and methods are described in the next section. Signal and image processing algorithms are described in Section 3. In Section 4 we will analyse our results and the influence of the target size, distance and camera type on them. Finally, the main conclusions are outlined.

\section{2.- MATERIAL AND METHODS}

\section{1.- EXPERIMENTAL SETUP}

The experiment was carried out on a pedestrian bridge located in Elche, a town in the south east of Spain. The bridge has a precast concrete deck and a metallic railing with a triangular design that could be part of the structural part of the bridge. Also, it is simply supported in one single span of $60 \mathrm{~m}$. This bridge is used to cross over a channel containing a small riverbed. The channel has been designed to prevent floods due to seasonal torrential rains that occur in the Mediterranean coast. Since these events only occur rarely, the riverbed and the channel have been adapted to allow different recreation activities. Therefore, the bridge offers a clean platform on the river bed with easy access for attaching a reference and for setting measuring devices underneath (reference measurements) at a distance of $9.5 \mathrm{~m}$. Fig. 1 shows a general view of the bridge.

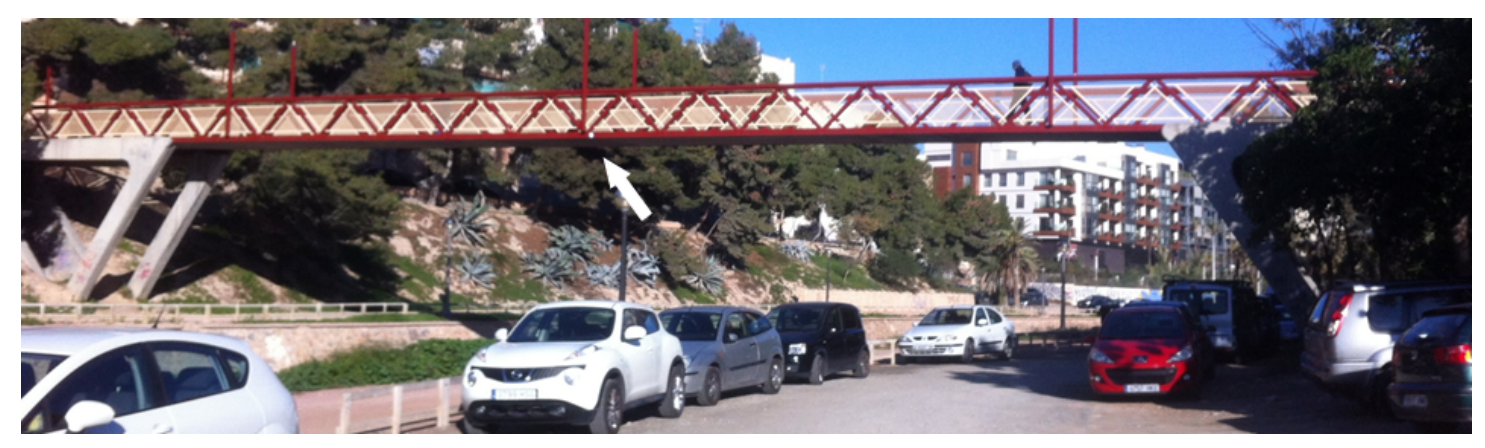


Fig. 1 Footbridge selected for the experiments. A white arrow is showing the target location

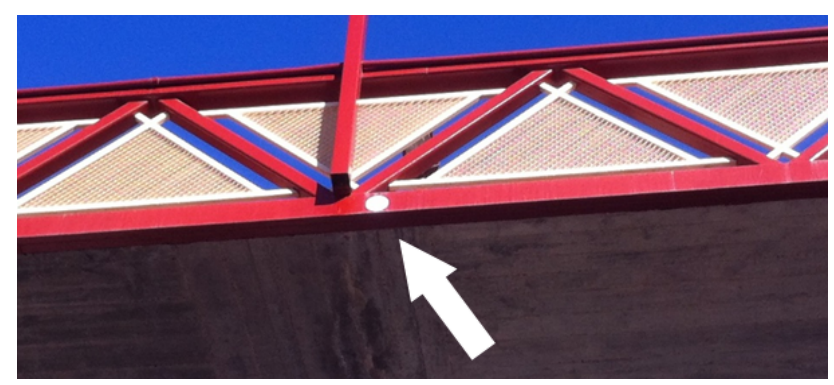

Fig. 2 Detail of the circular white target located in the middle span. A white arrow is pointing the target

In order to impose an oscillating load over the deck, two different types of actions were tested on the bridge: a swinging movement imposed by one person located in the middle span and in the centre of the deck, and an oscillatory movement caused by a person running along the bridge. In the case of the swinging movement the tester tried to adapt the excitation to the resonance frequency of the bridge, in order to make a bigger and clearer movement than those obtained with other swinging frequencies.

A circular target of $12 \mathrm{~cm}$ of diameter was attached to a bridge side in the middle span (see Fig. 1 and Fig.2). The vertical movement of the deck can be measured by tracking the displacement of this target with the image processing technique discussed in the introduction. Video sequences were acquired with two different systems. One of them was a Casio Exilim HS EX-ZR1000 camera working at 120 frames per second; for that temporal resolution, the frame size obtained is $640 \mathrm{x} 480 \mathrm{px}$. The other camera was a Basler acA640-120gc with a frame size of $658 \mathrm{x} 492 \mathrm{px}$ and a temporal resolution of 100 frames per second.

For both cameras the spatial resolution is relatively small so maximizing the $\mathrm{px} / \mathrm{mm}$ rate is needed in order to have a larger target size on the camera sensor. As we have a good natural illumination, we choose to use a telescope instead as a telephoto lens. The advantage of a telescope is that, for the same magnification, its price is much lower than a telephoto lens. Additionally, the telescope tripod adds stability to the system and the possibilities of assembling between any camera and a 
telescope are higher than between a video camera and a telephoto. Usually the telephoto is specifically designed for a particular still camera and the link with video cameras is difficult and does not give the best image.

Therefore, to increase the spatial resolution a Sky Watcher refractor telescope was used. The telescope has a diameter of $102 \mathrm{~mm}$ and a focal length of $500 \mathrm{~mm}$. It was mounted in a stainless tripod with a leg diameter of 1.75 ". On the one hand, adaptation of the Casio camera to the telescope requires of a digiscoping adapter, which is common in ornithology and astrophotography. The maximum optical zoom allowed for this camera (12.5x) was used. On the other hand, the Basler camera was directly assembled to the telescope using a metallic tube and without any lens, so the image was formed directly in the naked CCD. Fig. 3 shows the setup for Casio and Basler cameras with the telescope.

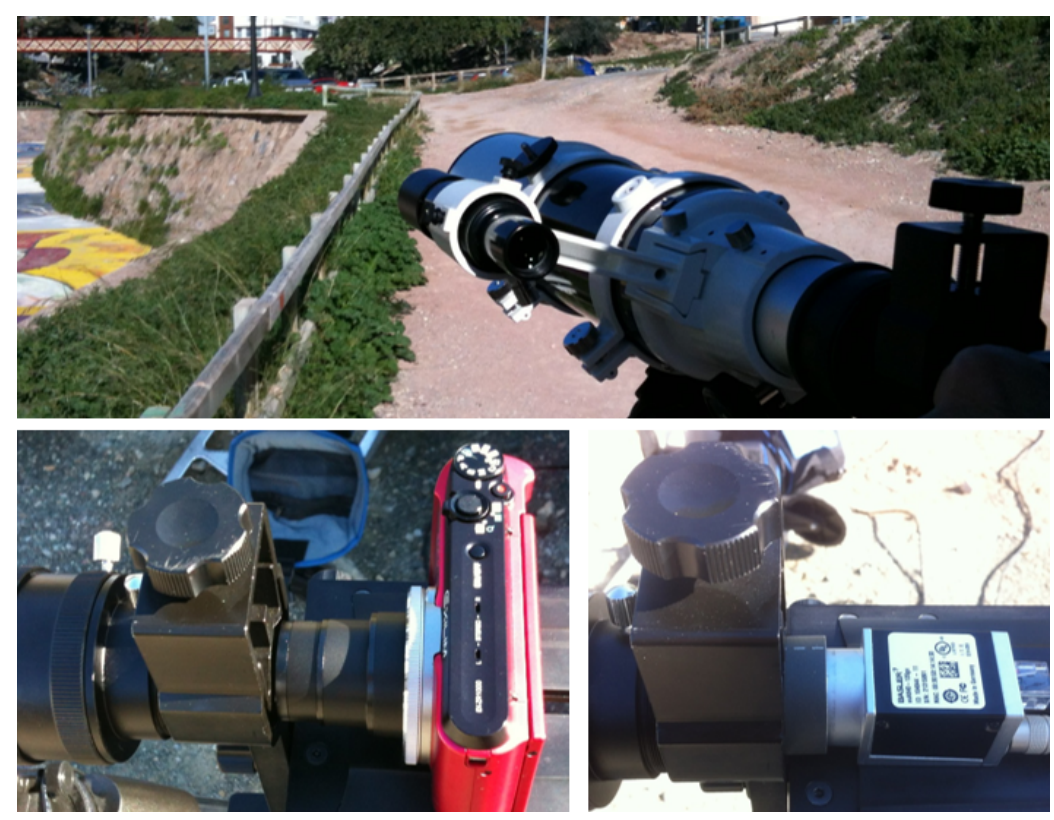

Fig. 3 General view of the set up recording at $200 \mathrm{~m}$ (upper side); detail of the connection between Casio camera and telescope (lower and left side); detail of the connection between Basler camera and telescope (lower and right side)

To assess the variations in the error as a function of the distance of measurement, four different tests were performed at distances from $50 \mathrm{~m}$ to $200 \mathrm{~m}$ with a stepped increase of $50 \mathrm{~m}$. For each 
distance both devices were used (Casio and Basler cameras), and both oscillating loads were used, thus having a total of sixteen recorded videos.

In order to determine the error in each measurement, a radar sensor was used simultaneously with the video capture. This radar sensor is an industrially engineered microwave interferometer used in the last decade in several vibration testing studies. An exhaustive description of the functioning principle was done by Gentile \& Cabboi [15]; for examples of applications to different structures testing, see: [16], [17], [18].

For the readers' convenience we recall that a microwave interferometer, as the one used for validating the results obtained in this experimental test, is able to provide the relative displacement of a target with respect to the radar, along the radar line of sight (LOS). This radar sensor has been used for the testing of several structures contributing to the modal analysis and providing estimates of the displacement with submillimeter accuracy. The accuracy is strongly related to the experimental conditions, especially to the signal to noise ratio [19]. The used radar is an IbisS system, marketed by Ids spa (Italy); its main characteristics are resumed in table1. To allow a comparison between the radar measurement and the investigated technique it is important to understand the geometry of the radar measurement. With respect to optical system, the field of view is given by the characteristics of the antenna and the other radar parameters. In this case, as shown in Fig. 4, the area illuminated by the radar corresponds to the intersection between the solid angle given by the antenna field of view and the deck, resulting in an elliptically shaped area.

Table 1: radar characteristics

\begin{tabular}{|l|c|}
\hline \multicolumn{2}{|c|}{ Ibis-S system characteristics } \\
\hline Operating frequency & $17.2 \mathrm{GHz}(\mathrm{Ku}$ band) \\
\hline Minimum range resolution & $0.5 \mathrm{~m}$ \\
\hline Antenna Field of View (FOV) & $0.314 \mathrm{rad} \times 0.66 \mathrm{rad}$ \\
\hline Maximum sampling frequency & $200 \mathrm{~Hz}$ \\
\hline
\end{tabular}


The radar was located on the riverbed under the bridge, at the middle point of the transversal width of the deck (Fig. 4 and 5). Using this geometry the part of the deck illuminated is practically at the same distance from the radar sensor line of sight and hence results as a single target. That gives a very clear radar response and the certainty that the radar is measuring the vertical movement of the deck and not a projection. It is worth noting that the radar sensor samples a portion of the deck providing a single value of its displacement. Therefore, the radar was used as a single point interferometer.
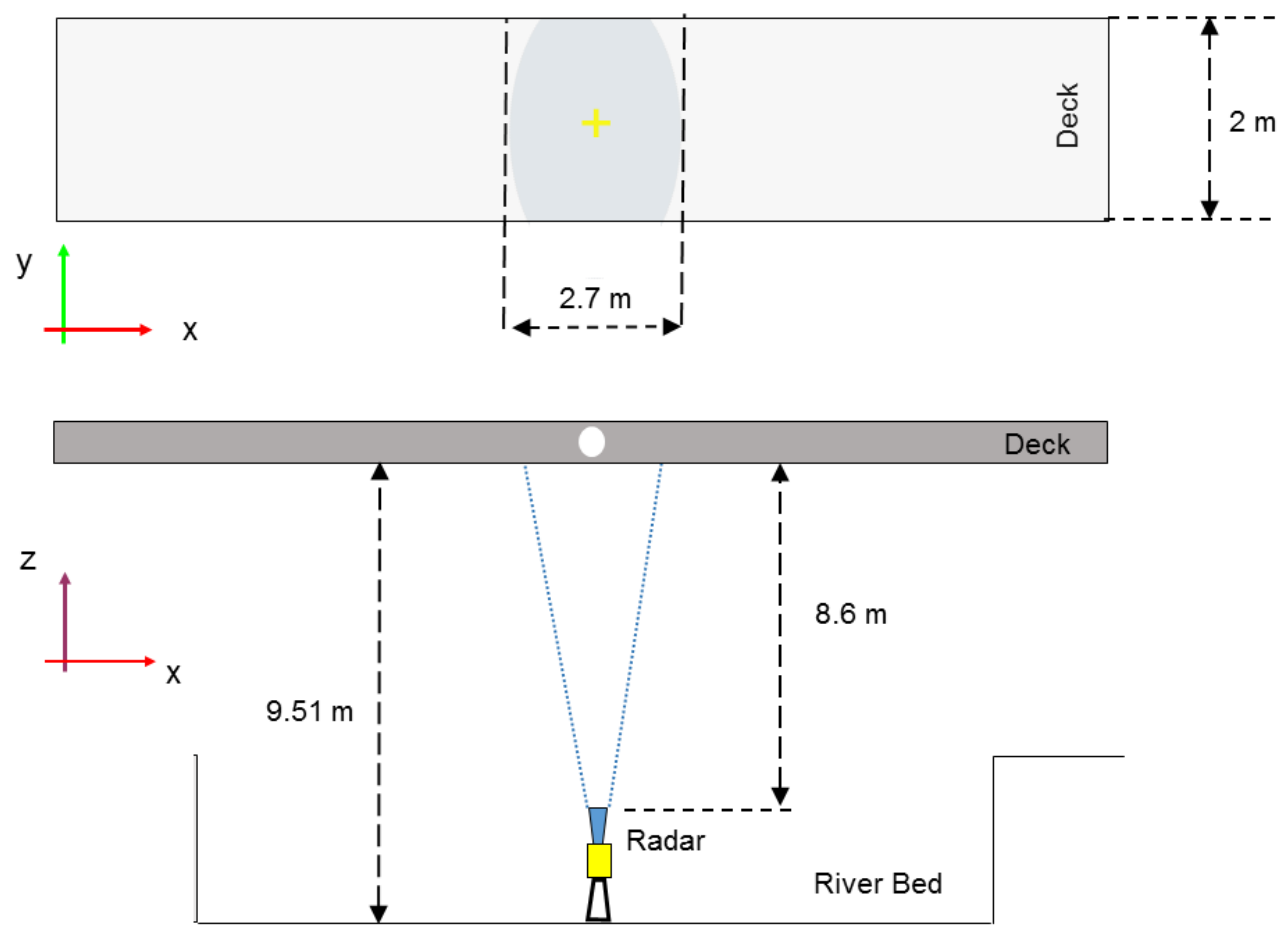

Fig. 4 Scheme of the radar acquisition geometry. The white circle represents the cameras target. 


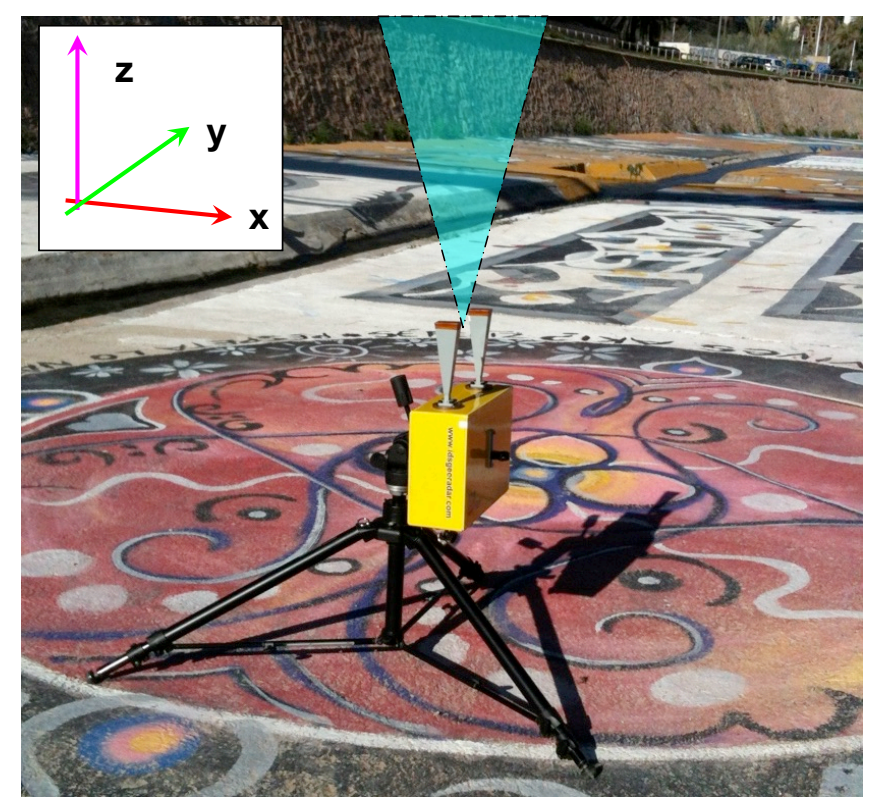

Fig. 5 Picture of the radar interferometer located in the bed of the river and pointing to the bottom part of the deck. A schematic representation of the antenna field of view and the 3-D axis reference are also included.

Bearing in mind that the excitation is done by one person and not mechanically (e.g using a vibrodyne), the amplitude given in different excitations is not strictly under control. Therefore, variations in error due to different amplitudes will be mixed with variations due to different distances or devices. In order to have information about the influence of amplitude in the error, an additional Casio camera was located on the riverbed, at $12 \mathrm{~m}$ from the deck. Due to its proximity to the deck the built-in camera objective was used and the use of a telescope was not necessary. Therefore, all measurements were registered in triplicate: with the radar, a close camera and one of the distant cameras with the telescope. Table 2 resumes the setup used during all the measurements.

Table 2. Excitations, distances and devices used in the experiment

\begin{tabular}{|l|c|}
\hline \multicolumn{2}{|c|}{ Combination of excitations, devices and distances } \\
\hline Excitation type & Swinging / Running \\
\hline
\end{tabular}




\begin{tabular}{|l|c|c|c|}
\hline Device & Casio + Telescope / Basler + Telescope & Casio & Interferometer \\
\hline Distances & $50 \mathrm{~m} / 100 \mathrm{~m} / 150 \mathrm{~m} / 200 \mathrm{~m}$ & $12 \mathrm{~m}$ & $8.6 \mathrm{~m}$ \\
\hline
\end{tabular}

\section{2.- IMAGE AND SIGNAL PROCESSING}

The vertical movement of the deck was obtained by detecting and tracking the movement of the circular target located in the deck. Since the optical axis of the camera is not perpendicular to the target, the perspective will deform its shape. Therefore, its appearance will not be a circle but an ellipse with changing axes depending on the camera locations. The measurement done is then a projection of the real vertical movement, but change from $\mathrm{px}$ to $\mathrm{mm}$ will assign the correct distance and the projection is compensated. Target tracking is accomplished by an automatic algorithm that searches and fits one ellipse shape in every image. This algorithm was already used in previous works, in which its accuracy was checked [8]. Also, in that work it is demonstrated that this procedure is able to detect movements with subpixel accuracy thus increasing the final resolution of the method. The center of the ellipse is selected as reference point to determine the movement of the deck.

With the signal obtained from the radar and the cameras, the vibration frequency of the bridge has also been analyzed. Sampling frequency was $98.04 \mathrm{~Hz}, 100 \mathrm{~Hz}$ and $120 \mathrm{~Hz}$ for radar, Basler camera and Casio camera, respectively. As the main frequency of the deck is around $3 \mathrm{~Hz}$, the sampling frequencies for the three devices used are able to record the real frequency of the bridge without aliasing effects. Since signals are obtained from different devices and procedures, some signal pre-processing is needed in order to allow their comparison. Therefore, all signals where resampled to $100 \mathrm{~Hz}$. A linear detrend was applied to all signals in order to remove the zero order in the Fourier transform. Finally, signals were normalized to the same energy value in order to allow direct comparison.

In Fig. 6 we represent the Fourier spectrum from both signals. The representation has been limited up to $5 \mathrm{~Hz}$ since no relevant information was found beyond this value. From the Fourier spectrum 
of both signals it is clear that the frequency content of the response is narrow-banded.A low frequency component can be observed both in the signal from the camera and the radar, although in this last device appears very much attenuated. In any case, this vibration may add a small modulation that will not affect very much to the result.

The signal to noise ratio of the radar measurements is very high $(>80 \mathrm{db})$ due to the short distance between the radar and the target, and the optimum observation geometry, i.e. the radar LOS is perpendicular to the reflecting surface (the deck). In this case the sensor is able to provide the best accuracy, of the order of tens of microns. The signal from the camera is expected to be noisier than that from the radar since its derivation is indirect and requires more steps, thus combining and increasing the propagated errors. Therefore, to reduce the signal noise, a bandpass Butterworth filter, order 3 , was used with a passband of $0.25 \mathrm{~Hz}$ each side to the main frequency, transition band of $1.5 \mathrm{~Hz}$ each side and attenuation of $3 \mathrm{~dB}$ and $40 \mathrm{~dB}$ in passband and eliminated band, respectively (Fig. 6). This filter was applied to both pre-processed signals, from cameras and from the interferometer, to allow the comparison of amplitudes in the same conditions.
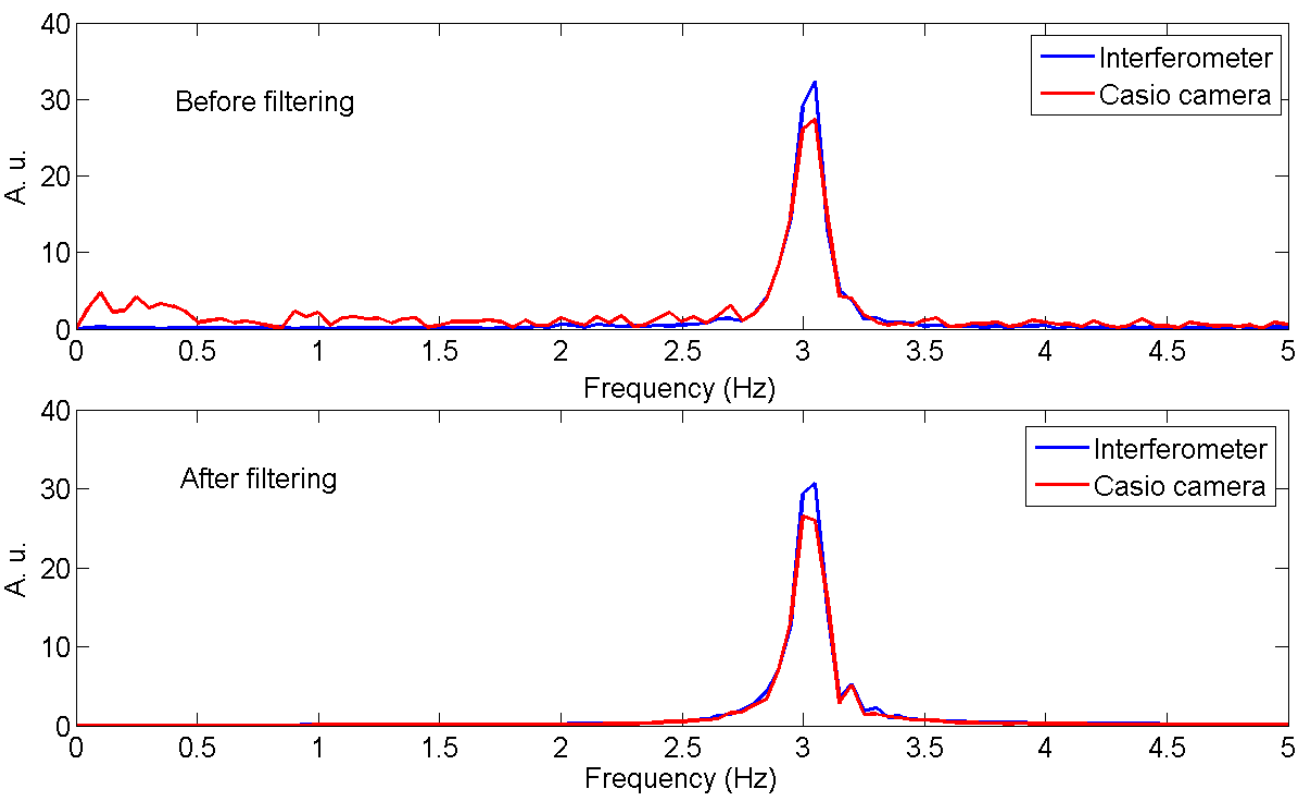

Fig. 6 Fourier transform of signal from interferometer and from Casio camera for the case of swinging recorded with Casio and telescope from $200 \mathrm{~m}$, before filtering (upper part) and after filtering (bottom part) 


\section{3.- RESULTS}

Before the application of the bandpass filter, all signals from cameras and interferometer show a main frequency of $3.0 \mathrm{~Hz}$, as the main frequency of the signal (Fig. 6 upper part). The spectra obtained from the cameras is noisier, with a secondary wide lobe in the range $0-0.5 \mathrm{~Hz}$. Since the energies are normalized, the main peak in the camera is less energetic than the equivalent one obtained from the radar. The noise and low frequency peak may be due to the camera support. The optical system is lighter than the radar, so it is more sensitive to the wind, mechanical drifts and uneven dilation of the tripod legs due to the sun. Also, derivation of the signal is done through indirect calculations thus adding calculation noise that is not present in direct measurements.

In any case, coincidence of the main lobe obtained from both methods is very good both in position and in shape. Although figure 6 only shows one particular case, the analysis and results are extensible to all the measurements done.

In Fig. 7 we show the time domain of the signal in Fig. 6 (bottom part; after filtering). We can see the good coincidence between them, although signal from camera shows a smaller amplitude than signal from radar. That difference in amplitude is due to the different total energy between signals. As normalization was done before filtering, some energy in signal from camera is wasted in low frequency and that leads to a smaller main peak than the main peak in signal from radar, after normalization. After filtering, low frequencies are eliminated and, since no renormalization was applied, total energy of signals is different in the considered interval thus leading to a smaller amplitude in the time domain. Nevertheless the difference in amplitude between signals is analyzed in the following sections and it is found that it is smaller than $10 \%$ in average. This discussion is also extensible to all the cases analyzed. 


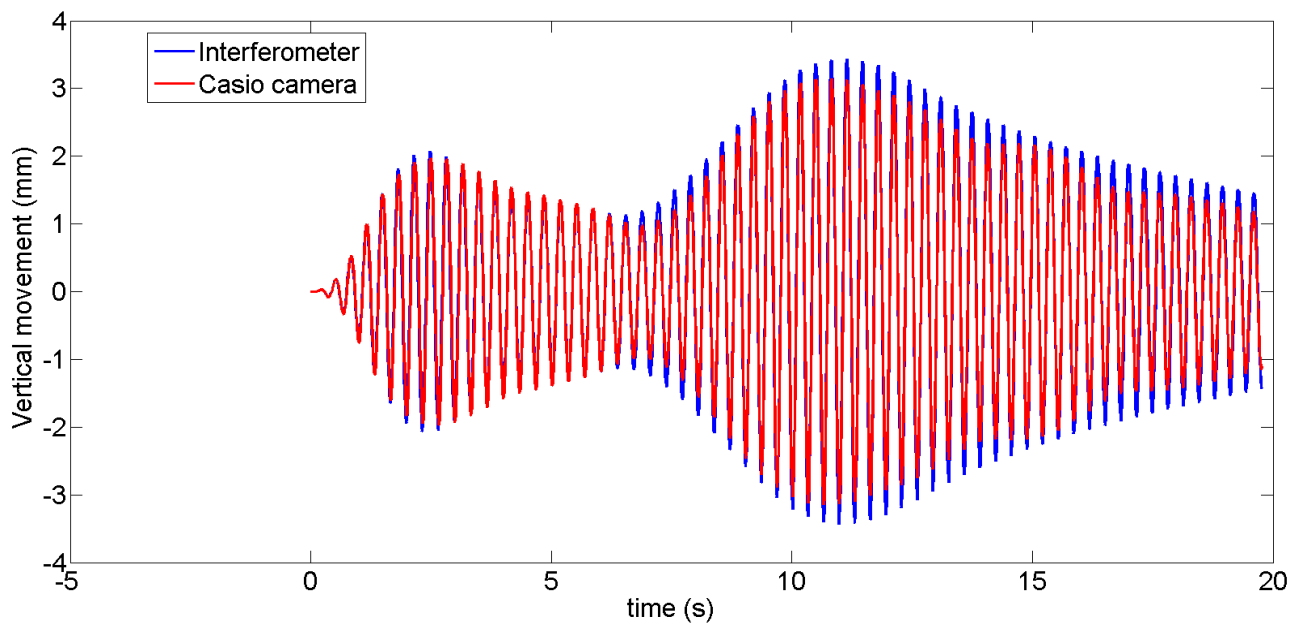

Fig. 7 Vertical movement obtained from interferometer and Casio camera for the case of swinging recorded with Casio and telescope from $200 \mathrm{~m}$ after filtering

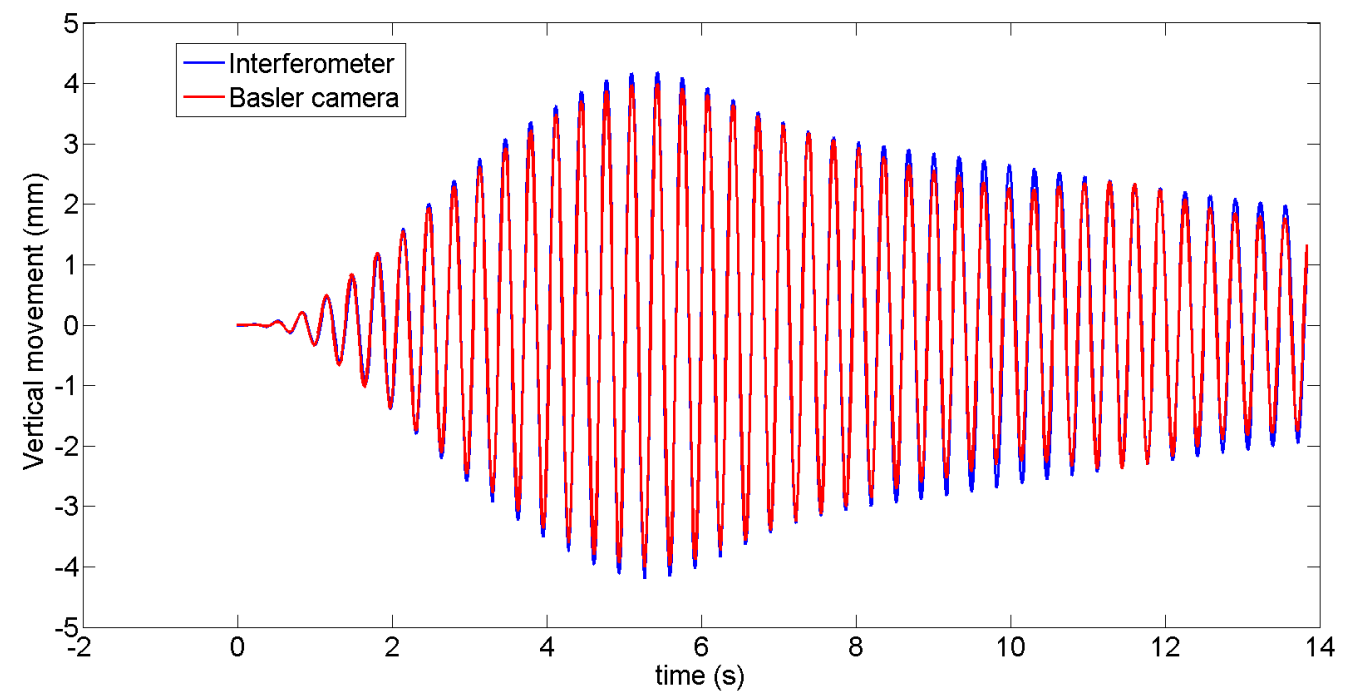

Fig. 8 Vertical movement obtained from interferometer and Basler camera for the case of running recorded with Basler and telescope from $200 \mathrm{~m}$ after filtering

This difference between the amplitudes seems to be higher for Casio camera than for Basler camera (Fig. 8). This fact is checked for the rest of signals and, as it will be shown below, the Basler camera give better results than Casio camera. Additionally, between cameras and interferometer there may be differences due to the different used techniques. The image 
processing method reconstructs the signal by tracking a target in the lateral side of the bridge. As previously anticipated, the radar interferometer, takes a single value for the portion of the area illuminated underneath the deck and the acquired data consists of a temporal $1 \mathrm{D}$ displacement history. Since the measurement point and the signal construction process are completely different, small discrepancies in the signal are expected.

To analyze these discrepancies, signals from radar have been taken as the references and the differences with respect to the signal obtained through the camera have been analyzed. Maximum value and standard deviation of the results have been calculated. Relative error was obtained by dividing the standard deviation by the maximum amplitude of the reference signal (from the radar). These values for all experiments are shown in tables 3 and 4 for results obtained from camera Casio located at $12 \mathrm{~m}$ and for cameras Casio and Basler located at different distances, respectively.

Table 3. Results for Casio camera located at $12 \mathrm{~m}$

\begin{tabular}{|c|c|c|c|c|c|c|c|}
\hline Device & \multicolumn{7}{|c|}{ CASIO } \\
\hline Type of bridge excitation & \multicolumn{7}{|c|}{ SWINGING } \\
\hline Distance to target & \multicolumn{7}{|c|}{$12 \mathrm{~m}$} \\
\hline Target diameter (px) & 37.76 & 37.76 & 37.5 & 37.48 & 37.55 & 37.51 & 37.45 \\
\hline $\begin{array}{l}\text { Max. Amplitude interferometer } \\
\text { (mm) }\end{array}$ & 0.98 & 0.38 & 6.55 & 6.26 & 2.60 & 3.43 & 0.69 \\
\hline $\begin{array}{l}\text { Max. Amplitude interferometer } \\
\text { (px) }\end{array}$ & 0.31 & 0.12 & 2.05 & 1.96 & 0.81 & 1.07 & 0.22 \\
\hline Máx. absolute error (mm) & 0.12 & 0.05 & 0.77 & 0.47 & 0.49 & 0.39 & 0.14 \\
\hline Máx. absolute error (px) & 0.04 & 0.02 & 0.24 & 0.15 & 0.15 & 0.12 & 0.04 \\
\hline $\begin{array}{l}\text { Standard deviation of signal } \\
\text { difference }(\mathrm{mm}) *\end{array}$ & 0.06 & 0.02 & 0.31 & 0.26 & 0.18 & 0.15 & 0.06 \\
\hline $\begin{array}{l}\text { Standard deviation of signal } \\
\text { difference }(p x) *\end{array}$ & 0.02 & 0.01 & 0.10 & 0.08 & 0.06 & 0.05 & 0.02 \\
\hline Relative error (Std/Max Amp.) & 0.06 & 0.05 & 0.05 & 0.04 & 0.07 & 0.04 & 0.09 \\
\hline Type of bridge excitation & \multicolumn{7}{|c|}{ RUNNING } \\
\hline
\end{tabular}




\begin{tabular}{|l|c|c|c|c|c|c|c|c|}
\hline Target diameter (px) & 37.60 & 37.51 & 37.53 & 37.49 & 37.62 & 37.56 & 37.46 & 37.51 \\
\hline Max. Amplitude interferometer & 3.40 & 4.65 & 3.51 & 0.45 & 2.77 & 3.60 & 5.38 & 4.19 \\
(mm) & & & & & & & & \\
\hline Max. Amplitude interferometer & 1.07 & 1.45 & 1.10 & 0.14 & 0.87 & 1.13 & 1.68 & 1.31 \\
\hline Máx. absolute error (mm) & 0.42 & 0.36 & 0.33 & 0.06 & 0.34 & 0.58 & 0.55 & 0.35 \\
\hline $\begin{array}{l}\text { Máx. absolute error (px) } \\
\text { Standard deviation of signal }\end{array}$ & 0.13 & 0.11 & 0.10 & 0.02 & 0.11 & 0.18 & 0.17 & 0.11 \\
difference (mm) * & & 0.14 & 0.19 & 0.03 & 0.15 & 0.24 & 0.27 & 0.21 \\
\hline Standard deviation of signal & 0.04 & 0.04 & 0.06 & 0.01 & 0.05 & 0.07 & 0.08 & 0.07 \\
difference (px) * & & & & & & & & 0.05 \\
\hline Relative error (Std/Max Amp.) & 0.04 & 0.03 & 0.05 & 0.06 & 0.05 & 0.06 & 0.05 & 0.05 \\
\hline
\end{tabular}

* Mean value of signal difference is 0

Table 4. Results for Casio and Basler cameras located at different distances

\begin{tabular}{|c|c|c|c|c|c|c|c|c|}
\hline Device & \multicolumn{4}{|c|}{ CASIO + TELESCOPE } & \multicolumn{4}{|c|}{ BASLER + TELESCOPE } \\
\hline Distance to target & $50 \mathrm{~m}$ & $100 \mathrm{~m}$ & $150 \mathrm{~m}$ & 200m & $50 \mathrm{~m}$ & $100 \mathrm{~m}$ & $150 \mathrm{~m}$ & $200 \mathrm{~m}$ \\
\hline Type of bridge excitation & \multicolumn{8}{|c|}{ SWINGING } \\
\hline Target diameter (px) & 132.89 & 58.93 & 45.48 & 31.25 & 266.81 & 115.83 & 84.02 & 58.95 \\
\hline $\begin{array}{l}\text { Max. Amplitude interferometer } \\
(\mathrm{mm})\end{array}$ & 0.98 & 6.46 & 6.26 & 3.43 & 3.40 & 3.51 & 2.77 & 5.38 \\
\hline $\begin{array}{l}\text { Max. Amplitude interferometer } \\
\text { (px) }\end{array}$ & 1.09 & 3.17 & 2.37 & 0.89 & 7.56 & 3.39 & 1.94 & 2.64 \\
\hline Máx. absolute error (mm) & 0.07 & 0.76 & 0.87 & 0.48 & 0.13 & 0.16 & 0.32 & 0.39 \\
\hline Máx. absolute error (px) & 0.08 & 0.37 & 0.33 & 0.12 & 0.30 & 0.16 & 0.22 & 0.19 \\
\hline $\begin{array}{l}\text { Standard deviation of signal } \\
\text { difference }(\mathrm{mm}) *\end{array}$ & 0.03 & 0.32 & 0.45 & 0.20 & 0.06 & 0.07 & 0.12 & 0.14 \\
\hline $\begin{array}{l}\text { Standard deviation of signal } \\
\text { difference }(\mathrm{px}) *\end{array}$ & 0.03 & 0.16 & 0.17 & 0.05 & 0.14 & 0.07 & 0.08 & 0.07 \\
\hline Relative error (Std/Max Amp.) & 0.03 & 0.05 & 0.07 & 0.06 & 0.02 & 0.02 & 0.04 & 0.03 \\
\hline Type of bridge excitation & & & & RUN & JING & & & \\
\hline Target diameter (px) & 132.97 & 59.27 & 45.40 & 31.25 & 268.54 & 115.83 & 84.17 & 58.93 \\
\hline
\end{tabular}




\begin{tabular}{|l|c|c|c|c|c|c|c|c|}
\hline Max. Amplitude interferometer & 0.38 & 2.54 & 2.60 & 0.69 & 4.66 & 0.45 & 3.60 & 4.19 \\
(mm) & & & & & & & & \\
\hline Max. Amplitude interferometer & 0.42 & 1.25 & 0.98 & 0.18 & 10.43 & 0.44 & 2.53 & 2.06 \\
\hline Máx. absolute error (mm) & 0.04 & 0.24 & 0.50 & 0.29 & 0.18 & 0.23 & 0.32 & 0.29 \\
\hline Máx. absolute error (px) & 0.05 & 0.12 & 0.19 & 0.08 & 0.41 & 0.23 & 0.22 & 0.14 \\
\hline Standard deviation of signal & 0.02 & 0.12 & 0.21 & 0.12 & 0.09 & 0.11 & 0.12 & 0.12 \\
difference (mm)* & & & & & & & & 0.06 \\
\hline Standard deviation of signal & 0.02 & 0.06 & 0.08 & 0.03 & 0.19 & 0.10 & 0.08 & 0.06 \\
difference (px) * & & & & & & & & 0.03 \\
\hline Relative error (Std/Max Amp.) & 0.04 & 0.05 & 0.08 & 0.18 & 0.02 & 0.23 & 0.03 & 0.03 \\
\hline
\end{tabular}

* Mean value of signal difference is 0

Graphical representation of these values gives a clearer view about the variations of the errors with respect to the rest of variables (signal amplitude, distance to target and target size). In principle, all variables could influence the error in measurement. Therefore, to isolate the influence of movement amplitude from other factors, the results obtained from camera at $12 \mathrm{~m}$ are analyzed, since in this case the distance to the target and the target size are constant for all measurements. As it was already discussed above, the distance to the target, besides to decrease the image resolution, increases the error due to the atmospheric distortion. Therefore, distance to target and image target diameter should be considered independents for an accurate analysis.

For Casio camera located at $12 \mathrm{~m}$, the representation of standard deviation versus the amplitude of signal is shown in Fig. 9. It is very easy to see that the error linearly increases with the amplitude. However, if we divide the standard deviation by its maximum amplitude, the representation (Fig. 10) shows that relative error slightly decrease with amplitude. In general, and with the exception of a single outlier, all relative errors are between 0.03 and 0.07 and the decrement is really soft for the same camera and the same distance to target. 


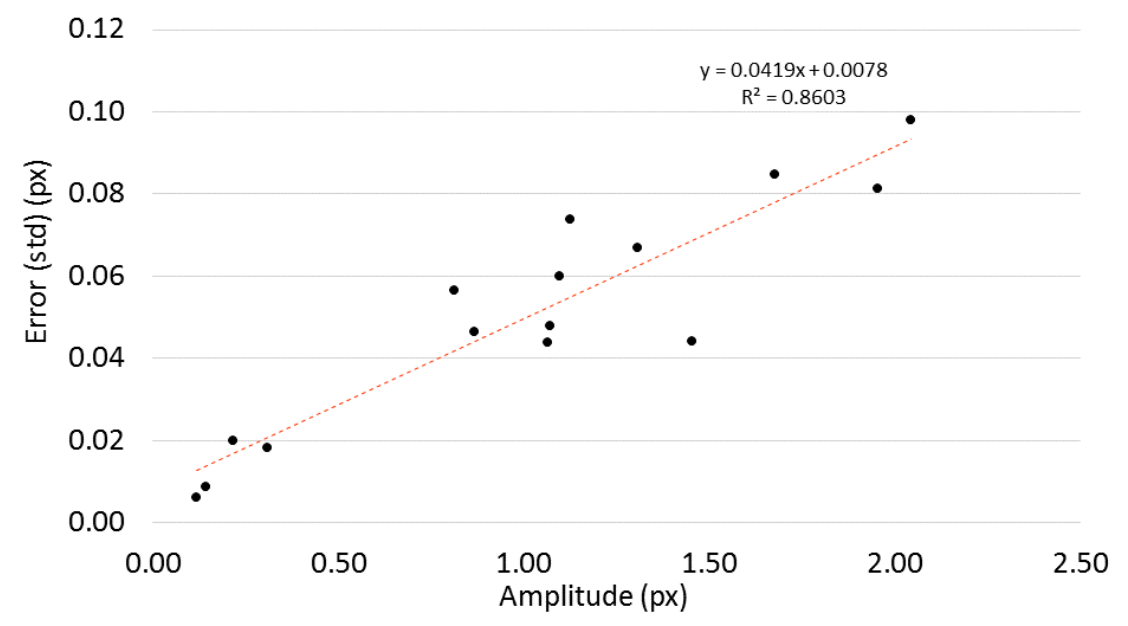

Fig. 9 Error (std) at $12 \mathrm{~m}$ vs. signal amplitude (px)

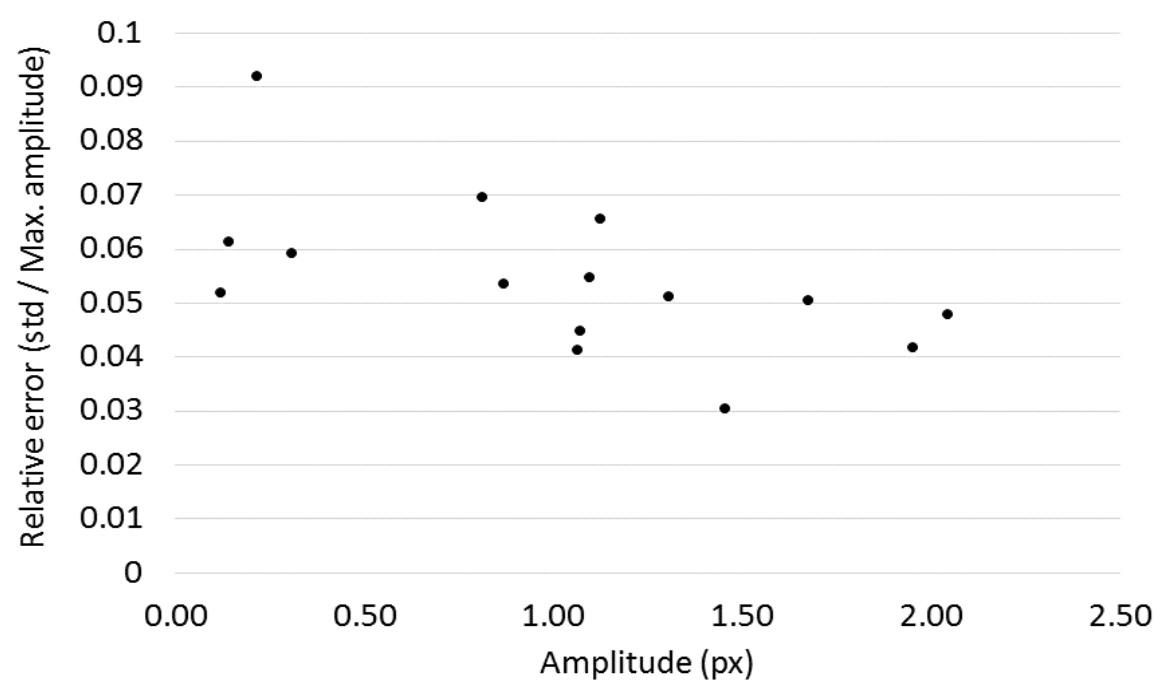

Fig. 10 Relative error (std/max. amplitude) at $12 \mathrm{~m}$ vs. signal amplitude (px)

For sequences obtained from cameras located at different distances to the target, relative error quickly decreases with the apparent target diameter in the frame (Fig. 11). This result was already observed in numerical simulations done by Mas et al. [8] and in experimental results obtained Busca et al. [7]. 


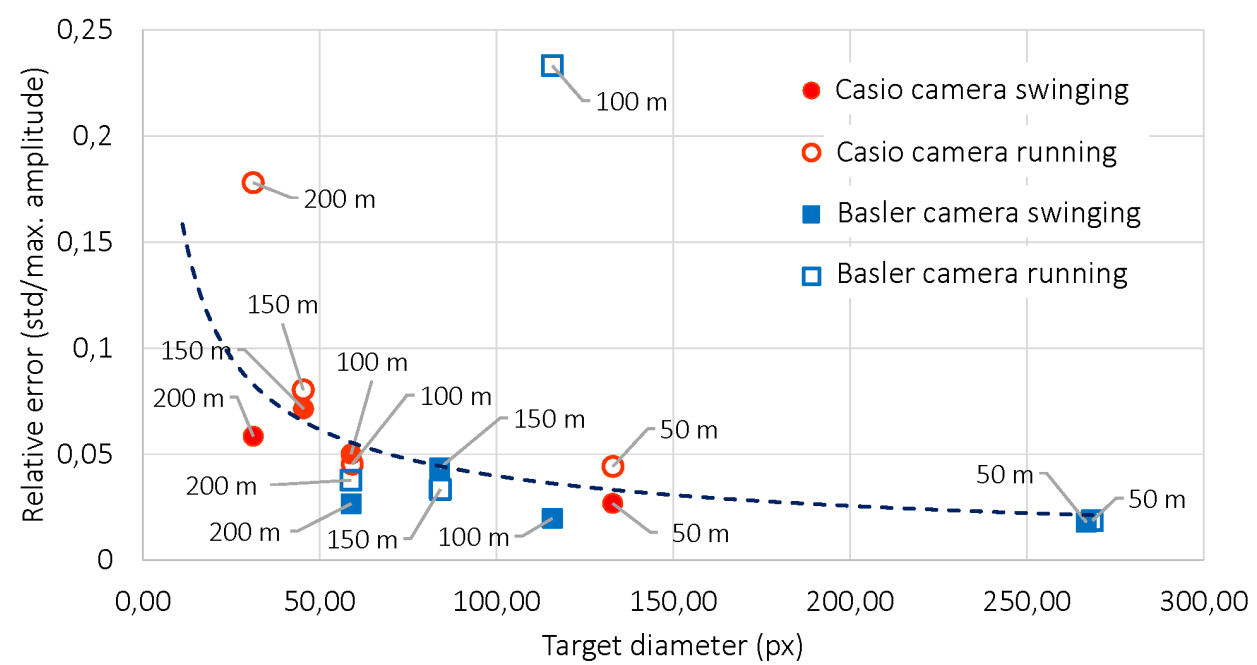

Fig. 11 Relative error (std/max. amplitude) vs. target diameter (px) for different distances, excitations and cameras. The distance to target is shown for each case in the data label. An exponential fitting curve has been obtained through the data

The variation of relative error with distance to target is shown in Fig. 12. In this plot we can also find one outlier, which corresponds to that described in Fig. 11; in both cases it represents the same measurement (Basler camera at $100 \mathrm{~m}$ for a running excitation). This anomalous result may be due to a camera displacement or some uncontrolled external factor. In any case, since this is the only anomalous result in the series, we can ignore this result without losing the validity of our results. There is also a point corresponding to the Casio camera (200 $\mathrm{m}$ and running excitation) that could also be an outlier. However, in this case the difference between this error and the following highest error is of 0.1 , while in the case of the Basler outlier that difference is 0.19 so we decided not excluding it from our analysis.

Errors from both cameras increase with distance to the target but this tendency is stronger for Casio camera than for Basler camera (Fig. 12). These results are consistent with those obtained from Fig. 11, because at closer distances the target diameter in the frame is larger and that leads to lower relative errors. However, at higher distances, errors coming from atmospheric dispersion, 
camera movements due to wind or even to ground vibrations become more important and may increase the relative error of the measurement.

To analyze the relative error variations due to movement amplitude variations, Fig. 13 is presented. It can be found again the outlier corresponding to Basler camera at $100 \mathrm{~m}$ for a running excitation. The trend is clearly decreasing with the movement amplitude. In fact, a potential law can be adjusted to these data, in a similar way it was done in Fig. 11.

It may be surprising that higher amplitudes are only recorded with the Basler camera, but this fact is due to the casual absence of larger movements when the experiment was registered with the Casio camera. Additionally, there is no evidence that lead to think that a large movement amplitude recorded by a Casio camera will give a higher relative error than a Basler camera.

Notice that, except for the outlier case (Basler running $100 \mathrm{~m}$ ) the relative error for Casio camera is always higher than that obtained from Basler camera, which is always below 0.05 . It is difficult to determine the reason for this error difference. Both cameras are small and were subjected by the digiscoping adaptor. There are some differences regarding the hardware and the operation modes. On the one hand, the Basler camera is a gray scale low-cost scientific device, so we can control all parameters (gain, exposition, etc.) and the sequences are saved without any compression or interpolation. On the other hand, the Casio camera is a consumer camera with predefined automatic capturing modes. The sensor is RGB, which implies that color images are not real but interpolated [20]. Additionally, the sequences are saved in AVI-JPEG format, which introduces a lossy compression in each frame. All these factors limit the image quality finally obtained, so the data calculated from the sequence have poorer quality and thus, may introduce larger errors in the signal.

Finally, we would like to remark that we could not find any trend regarding the type of excitation in the obtained relative error. 


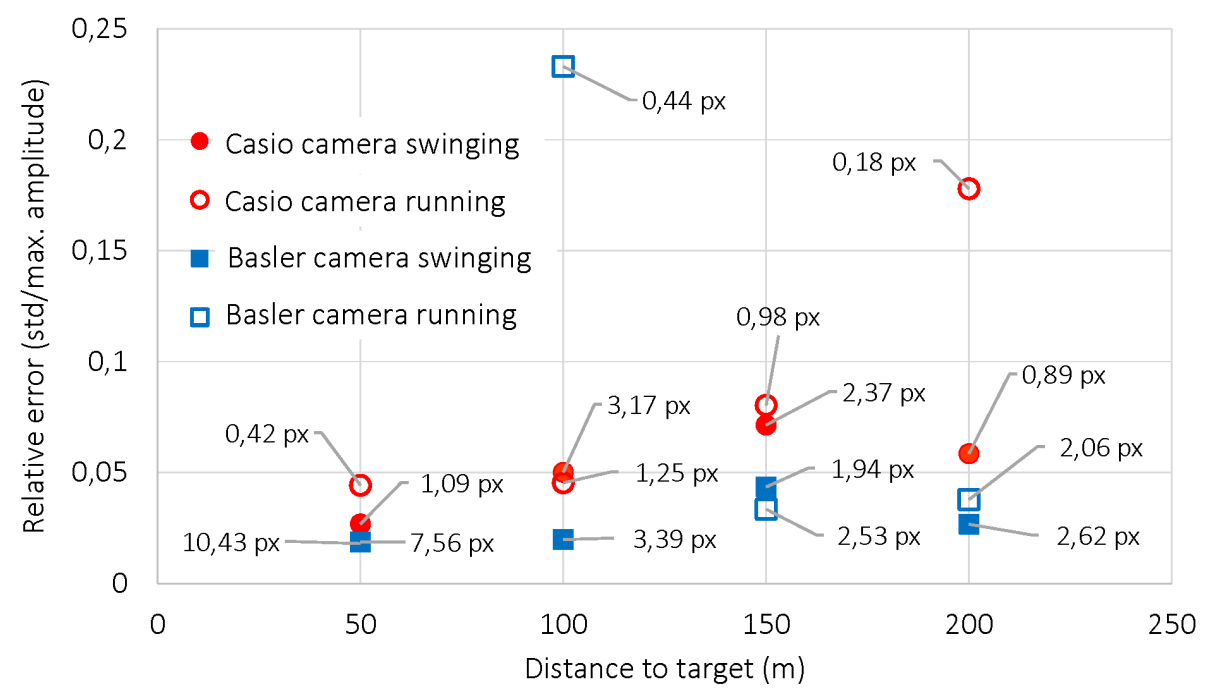

Fig. 12 Relative error (std/max. amplitude) vs. distance to the target (m) for different excitations and cameras. The maximum movement amplitude in px for each case is shown in each data label

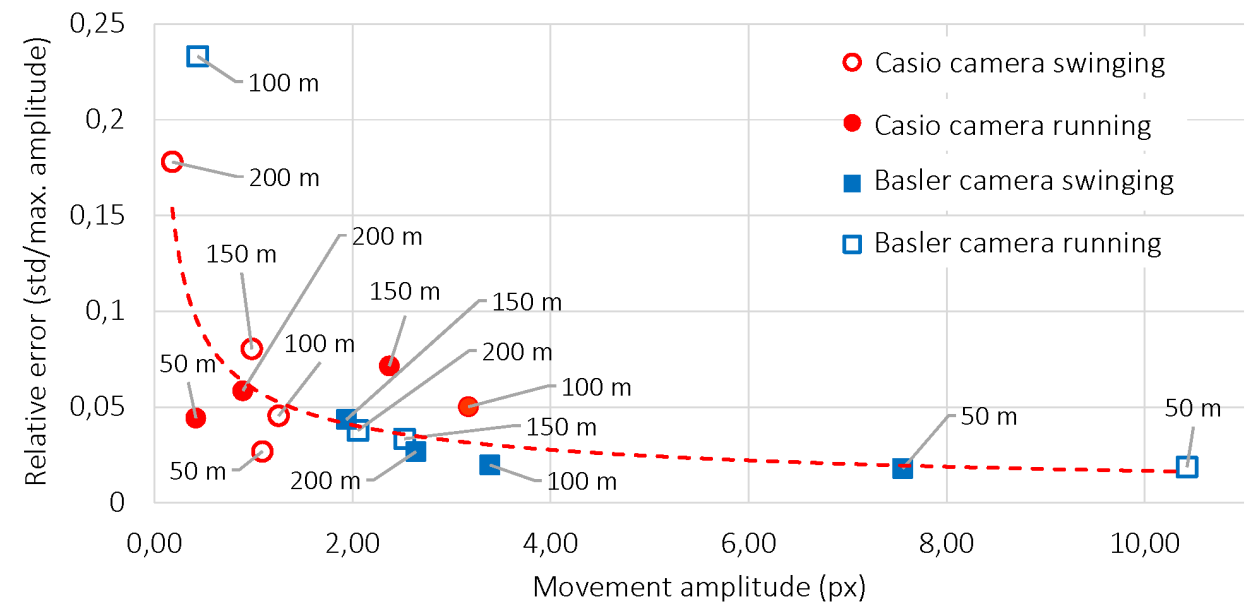

Fig 13 Relative error (std/max. amplitude) vs movement amplitude (px) for different target distances, excitations and cameras. The target distance for each case is shown in each data label. A potential law has been adjusted to data

\section{4.- CONCLUSIONS}

In this work a series of experiments have been carried out in which the oscillating movement of a bridge was monitored and the deck's deflection estimated using image processing methods based on template matching and least square fitting. To obtain a reliable reference of the 
displacement amplitude and oscillating frequency, the results have been compared with those obtained with a radar interferometer, whose accuracy was checked in previous works. Different cameras, distances and excitation types were checked and the relative error was determined by dividing the standard deviation of the signal subtraction (radar interferometer minus camera) by the maximum movement amplitude.

From the results we can conclude that, for all distances, target sizes and movement amplitudes, the relative error for Basler camera and telescope is smaller than 5\%, except for one outlier. That is to say, in the worst case, the standard deviation of absolute error was $0.12 \mathrm{~mm}$ for a movement amplitude of $2.77 \mathrm{~mm}$. For the Casio camera and telescope the relative error is, in all but one case, smaller than $10 \%$.

Results obtained from the same distance and with the same target diameter in the image show that the relative error slightly decreases with movement amplitude (Fig. 10). For different distances, in which the atmospheric distortion and the cameras movement have more importance, the relative error decreases (approximately following a potential law) with the movement amplitude (Fig. 13).

The variation of relative error due to the excitation type is not significant, for any of the analysis done in this study. Therefore, the trends obtained here for different amplitudes, target distances and target diameter in pixels include all points and make no difference between points related with different excitation types. That implies also that the way in which the excitation is done in the bridge is not relevant for the results and the results obtained will be true even with other excitation types.

Regarding the target diameter in the frame, the relative error exponentially decrease as the diameter increases (Fig. 11), as it was already observed in numerical analysis done by Mas et al. [8] and in the experiments done Busca et al. [7]. The rise in distance to target give an increase in relative error for both cameras, but for Casio camera that trend is stronger (Fig. 10). In this case the trend seems to be linear. 
In all cases we can observe that the Basler camera gives better results than Casio camera. That may be due to the compression algorithms that Casio introduces in every frame. The automatic modes of the Casio camera could also be not optimal thus producing imprecise results.

From this work, it is clear that the camera selection, even in the low end segment, is of fundamental importance to obtain accurate results. Although its cost is similar, the technical specifications may show that the Casio camera is a better acquiring device. Nevertheless the Basler camera permits optimization of the parameters as well as avoiding compression algorithms in the acquired sequence. Although these cameras are harder to use, since they require some technical knowledge, the quality of results are worth the effort.

This being said, if one is only interested in a preliminary test and obtain rough measurement, results here also show that pocket cameras can be a reliable alternative to more sophisticated systems, even for high distances. It is true that the errors can be relatively high for some applications, but for the main interests that errors are reasonable taking into account the distance from the measurement point. Also, the frequency measurement is accurate and the device is cheap, small, manageable and widely available.

\section{REFERENCES}

[1] Wahbeh AM, Caffrey JP, Masri SF (2003) A vision-based approach for the direct measurement of displacements in vibrating systems. Smart Materials and Structures 12:785-794

[2] Lee JJ, Shinozuka M (2006) A vision-based system for remote sensing of bridge displacement. NDT\&E International 39:425-431

[3] Genturk B, Hossain K, Kapadia A, Labib E, Mo Y-L (2014) Use of digital image correlation technique in full-scale testing of prestressed concrete structures. Measurement 47:505-515

[4] Malesa M, Kujawinska M (2013) Deformation measurements by digital image correlation with automatic merging of data distributed in time. Applied Optics 52 (19):4681-4692 
[5] Verbruggen S, Aggelis DG, Tysmans T, Wastiels J (2014) Bending of beams externally reinforced with TRC and CFRP monitored by DIC and AE. Composite structures 112:113-121

[6] Shah SG,Chandra Kishen JM (2011) Fracture properties of concrete-concrete interfaces using digital image correlation. Experimental mechanics 51 (3):303-313

[7] Busca G, Cigada A, Mazzoleni P, Zappa E (2014) Vibration monitoring of multiple bridge points by means of a unique vision-based measuring system. Experimental mechanics 54:255271

[8] Mas D, Espinosa J, Roig AB, Ferrer B, Perez J, Illueca C (2012) Measurement of wide frequency range structural microvibrations with a pocket digital camera and sub-pixel techniques. Applied Optics 51(14):2664-2671

[9] Ferrer B, Acevedo P, Espinosa J, Mas D (2015) Targetless image-based method for measuring displacements and strains on concrete surfaces with a consumer camera. Construction and building materials 75:213-219

[10] Chien-Chou C, We-Hwa W, Hong-Zeng T, Chi-Ong C, Gwolong L (2015) Application of digital photogrammetry techniques in identifying the mode shape ratios of stay cables with multiple camcorders. Measurement 75:134-146

[11] Fukuda Y, Feng MQ, Narita Y, Kaneko S, Tanaka T (2013) Vision-based displacement sensor for monitoring dynamic response using robust object search algorithm. IEEE Sensors Journal 13 (12):4725-4732

[12] Tubbs RN (2003) Lucky exposures: Diffraction Limited Astronomical Imaging through the Atmosphere. Dissertation submitted in candidature for the degree of Doctor of Philosophy in the University of Cambridge. St Johns College Cambridge University

[13]https://www.meteoblue.com/es/tiempo/pronostico/seeing/alicante espa\%C3\%B1a 2521978 Accessed 30 January 2016 
[14] Roig AB, Espinosa J, Perez J, Ferrer B, Mas D (2015) Three-dimensional planar object tracking with sub-pixel accuracy Optik 126 (20):2684-2689

[15] Gentile C, Cabboi A (2015) Vibration-based structural health monitoring of stay cables by microwave remote sensing. Smart Structures and Systems 16 (2):263-280.

[16] Atzeni C, Bicci A, Dei D, Fratini M, Pieraccini M, (2010) Remote survey of the leaning tower of Pisa by interferometric sensing. IEEEGRSL 7 1:185-189

[17] Negulescu C, Luzi G, Crosetto M, Raucoules D, Roullé A, Monfort D, Pujades L, Colas B, Dewez T, (2013) Comparison of seismometer and radar measurements for the modal identification of civil engineering structures. Engineering Structures 51:10-22.

[18] Luzi G, Crosetto M, Cuevas-González M. (2014) A radar-based monitoring of the Collserola Tower (Barcelona). Mechanical Systems and Signal Processing 49:234-248.

[19] Coppi F, Gentile C, Ricci P (2010) A software tool for processing the displacement time series extracted from raw radar data. Proceedings of the Ninth International Conference on Vibration Measurements by Laser and Non-contact Techniques, AIP Conference Proceedings 1253, E.P.Tomasini (Ed.), Ancona, Italy pp 22-25

[20] https://en.wikipedia.org/wiki/Bayer filter Accessed 28 February 2016 